\title{
An Integrated Perspective of Food Consumption and Depression in Young Adulthood
}

\author{
Jaewon Lee M.S.W, PHD $^{1^{*}}$ and Jennifer Allen, M.S.W' \\ ${ }^{1}$ Assistant Professor, Department of Social Welfare, Inha University, South Korea \\ ${ }^{2}$ Doctoral Student, School of Social Work, Michigan State University, USA
}

\begin{abstract}
Gender differences in depression have long been examined in the mental health literature [1,2]. Among many diverse factors that influence depression in young adulthood, patterns of food consumption, which also differ by gender, could be one important determinant, particularly because young adults are more likely to eat unhealthy foods (such as fast foods or soft drinks/sodas with sugar) compared to other age groups (e.g., [3-8]). Lee and Allen's article explored the moderating effect of gender on the relationship between patterns of food consumption and depression in young adulthood [9].
\end{abstract}

Gender differences in depression have long been examined in the mental health literature [1,2]. Among many diverse factors that influence depression in young adulthood, patterns of food consumption, which also differ by gender, could be one important determinant, particularly because young adults are more likely to eat unhealthy foods (such as fast foods or soft drinks/sodas with sugar) compared to other age groups (e.g., [3-8]). Lee and Allen's article explored the moderating effect of gender on the relationship between patterns of food consumption and depression in young adulthood [9].

\section{A New Light on Depression and Food Con- sumption}

Interestingly, Lee and Allen's article showed that healthy food consumption (fruits and vegetables), but not unhealthy food consumption (fast food and soft drinks), was related to depression among young adults. This is a meaningful finding because unhealthy and healthy foods were included in a single model. Previous studies generally considered healthy and unhealthy food consumption separately (e.g., $[10,11])$. However, Lee and Allen's study considered four types of healthy and unhealthy foods. As young adults commonly consume unhealthy foods such as fast food and soft drinks, it is possible that unhealthy foods might not influence depression among young adults. Further, given young women's higher risk of depression, the moderating effect of gender implied that increased consumption of fruit is critical to reducing depression among young women. Recognizing the gender differences in depression and food consumption in young adulthood, Lee and Allen's study sheds light on future mental health and nutrition policies targeted toward young adults.

\section{Limitations and Future Directions}

Even though Lee and Allen's study contributes to the literature on nutrition and depression in young adulthood, some limitations should be noted. First, unhealthy and healthy foods were limited to four types, and future studies may consider additional food types such as snacks, meats, grains, etc. Second, young adulthood in this study included those aged 18 to 35 -years-old. However, the age range for young adulthood differs by study (e.g., 18-25, 18-30, and 18-34 years; $[8,12,13])$ and future studies should provide a rationale for determining the age range for young adulthood. Third, a theoretical framework using stress process theory, for example, may help explain the logic of Lee and Allen's article. Last, the causality of the findings cannot be determined due to the use of cross-sectional data. Future studies may benefit from approaches that allow for the interpretation of causality or longitudinal approaches to identify how the relationship between food consumption patterns and depression differ by gender over time.

\section{Conclusion}

Young adults' food consumption habits differ compared

*Corresponding author: Jaewon Lee, M.S.W, PhD, Assistant Professor, Department of Social Welfare, Inha University, 100 Inha-ro, Michuhol-gu, Incheon 22212, South Korea, Tel: +82-32860-9324, Fax: +82-32-863-3022

Accepted: November 19, 2020

Published online: November 19, 2020

Citation: Lee J, Allen J (2020) An Integrated Perspective of Food Consumption and Depression in Young Adulthood. J Depress Anxiety Disord 2(1):66-67 
to previous generations as they may easily access unhealthy foods such as junk foods. Changes in young adults' life may influence their mental health in the context of what types of food they consume. In particular, young women are at greater risk of depression than young men, so it is important to examine gender differences in this relationship. In this context, Lee and Allen's research sheds light on future policy and practice by considering gender differences in food consumption patterns and depression. For future studies exploring this relationship, we recommend a longitudinal approach that allows for causal inferences and considers the impact of gender differences over the life course.

\section{References}

1. Deborah S Hasin, Aaron L Sarvet, Jacquelyn L Meyers, et al. (2018) Epidemiology of adult DSM-5 major depressive disorder and its specifiers in the United States. JAMA Psychiatry 75: 336346.

2. National Institute of Mental Health (2019) Major depression.

3. Centers for Disease Control and Prevention (2017) Get the facts: Sugar-sweetened beverages and consumption.

4. Fryar CD, Hughes JP, Herrick KA, et al. (2018) NCHS data brief: Fast food consumption among adults in the United States, 20132016. Centers for Disease Control and Prevention.

5. Liu X, Yan Y, Li F, et al. (2016) Fruit and vegetable consumption and the risk of depression: A meta-analysis. Nutrition 32: 296302.
6. Mikolajcyzk RT, El Ansari W, Maxwell AE (2009) Food consumption frequency and perceived stress and depressive symptoms among students in three European countries. Nutr J 8: 31.

7. Morse KL, Driskell JA (2009) Observed sex differences in fast food consumption and beliefs of college students. Nutrition Research 29: 173-179.

8. Nour M, Sui Z, Grech A, et al. (2017) The fruit and vegetable intake of young Australian adults: A population perspective. Public Health Nutr 20: 2499-2512.

9. Lee J, Allen J (2020) Gender differences in healthy and unhealthy food consumption and its relationship with depression in young adulthood. Community Mental Health Journal.

10. Angelino D, Godos J, Ghelfi F, et al. (2019) Fruit and vegetable consumption and health outcomes: An umbrella review of observational studies. Int J Food Sci Nutr 70: 652-667.

11. Van der Horst K, Brunner TA, Siegrist M (2011) Fast food and take-away food consumption are associated with different lifestyle characteristics. Journal of Human Nutrition and Dietetics 24: 596-602.

12. Allgower A, Wardle J, Steptoe A (2001) Depressive symptoms, social support, and personal health behaviors in young men and women. Health Psychol 20: 223-227.

13. Gibb SJ, Ferguson DM, Horwood L (2010) Burden of psychiatric disorder in young adulthood and life outcomes at age 30 . The British Journal of Psychiatry 197: 122-127.

DOI: $10.36959 / 362 / 474$

Copyright: (C) 2020 Lee J, et al. This is an open-access article distributed under the terms of the Creative Commons Attribution License, which permits unrestricted use, distribution, and reproduction in any medium, provided the original author and source are credited. 